\title{
Квантово-каскадный лазер с выводом излучения через текстурированный слой
}

\author{
(C) A.В Бабичев ${ }^{1}$, E.C Колодезный ${ }^{1}$, А.Г Гладышев ${ }^{1}$, Д.В Денисов ${ }^{2}$, Г.В Вознюк ${ }^{3}$, М.И Митрофанов ${ }^{3}$, \\ Д.А Михайлов ${ }^{3}$, Д.В Чистяков ${ }^{3}$, Д.И Курицын ${ }^{4}$, В.В Дюделев ${ }^{3}$, С.О Слипченко ${ }^{3}$, \\ А.В Лютецкий ${ }^{3}$, В.П Евтихиев ${ }^{3}$, Л.Я Карачинский ${ }^{1,3,5}$, И.И Новиков ${ }^{1,3,5}$, \\ С.В Морозов ${ }^{4}$, Г.С Соколовский ${ }^{3}$, Н.А Пихтин ${ }^{3}$, А.Ю Егоров ${ }^{1}$ \\ ${ }^{1}$ Национальный исследовательский университет ИТМО, \\ 197101 Санкт-Петербург, Россия \\ ${ }^{2}$ Санкт-Петербургский государственный электротехнический университет „ЛЭТИ“ им. В.И. Ульянова (Ленина), \\ 197022 Санкт-Петербург, Россия \\ ${ }^{3}$ Физико-технический институт им. А.Ф. Иоффе Российской академии наук, \\ 194021 Санкт-Петербург, Россия \\ ${ }^{4}$ Институт фризики микроструктур Российской академии наук, \\ 603950 Нижний Новгород, Россия \\ ${ }^{5} \mathrm{OOO} \mathrm{„Коннектор} \mathrm{Оптикс“,}$ \\ 194292 Санкт-Петербург, Россия \\ E-mail: a.babichev@mail.ioffe.ru
}

Поступила в Редакцию 19 апреля 2021 г.

В окончательной редакции 27 апреля 2021 г.

Принята к публикации 27 апреля 2021 г.

\begin{abstract}
Представлены результаты исследований кольцевых квантово-каскадных лазеров с выводом излучения через текстурированный слой, сформированный в слоях верхней обкладки волновода методом сверхвысоковакуумного ионно-лучевого травления. Исследование распределения интенсивности излучения в зоне дальнего поля показывает, что сформированные окна, содержащие текстурированный слой, позволили осуществить направленный поверхностный вывод излучения из кольцевого лазера. Вывод излучения осуществляется в диапазоне углов $\sim(63-75)^{\circ}$, отсчитанных от нормали к плоскости резонатора.
\end{abstract}

Ключевые слова: сверхрешетки, квантово-каскадный лазер, эпитаксия, фосфид индия, кольцевой резонатор, текстурированный слой.

DOI: 10.21883/FTP.2021.11.51564.9670

\section{1. Введение}

К настоящему моменту достигнут значительный прогресс в области формирования поверхностно-излучающих квантово-каскадных лазеров (ККЛ) среднего инфракрасного (ИК) диапазона в конструкции с селективным кольцевым резонатором $[1,2]$. Для реализации вывода излучения через поверхность в квантово-каскадных лазерах на межподзонных переходах с ТМ поляризацией излучения используют дифракционные решетки 2-го порядка, сформированные вытравливанием областей в слоях верхней обкладки волновода $[3,4]$ или в специальных слоях InGaAs, расположенных вблизи активной области, с последующим заращиванием и формированием верхней обкладки волновода [5]. Угол вывода излучения по отношению к нормали к поверхности гетероструктуры определяется на основе выражения

$$
\alpha=\arcsin \left[n_{\mathrm{ef}} / n_{0}-\lambda /\left(\Lambda n_{0}\right)\right],
$$

где $n_{\mathrm{ef}}$ - эффективный показатель преломления для лазерного излучения, $n_{0}$ - показатель преломления окружающей среды, $\lambda$ - длина волны, $\Lambda$ - период дифракционной решетки [6].

Вместе с тем в последнее время особый интерес для вывода излучения представляют субволновые решетки.
На основе одномерных субволновых решеток сформированы антиотражающие покрытия для полосковых ККЛ [7]. Субволновые решетки являются частным случаем субволновых текстурированных слоев. Для их описания можно применить теорию эффективной среды, где текстурированный слой рассматривается как однородная среда с неким эффективным показателем преломления, определяемым фактором заполнения [8,9].

Использование субволновых антиотражающих покрытий на основе текстурированных поверхностей пирамидальной формы [8], где показатель преломления плавно уменьшается от показателя преломления полупроводника до показателя преломления воздуха [10], позволяет реализовать коэффициент отражения, близкий к нулю в широком диапазоне углов падения.

В настоящей работе представлены результаты по формированию и изучению характеристик поверхностноизлучающих ККЛ спектрального диапазона 7.5-8.0 мкм с кольцевым резонатором, где вывод излучения осуществляется через окна с текстурированным слоем.

\section{2. Эксперимент}

Гетероструктура ККЛ была выращена на подложке InP c ориентацией (001) компанией „Коннектор 

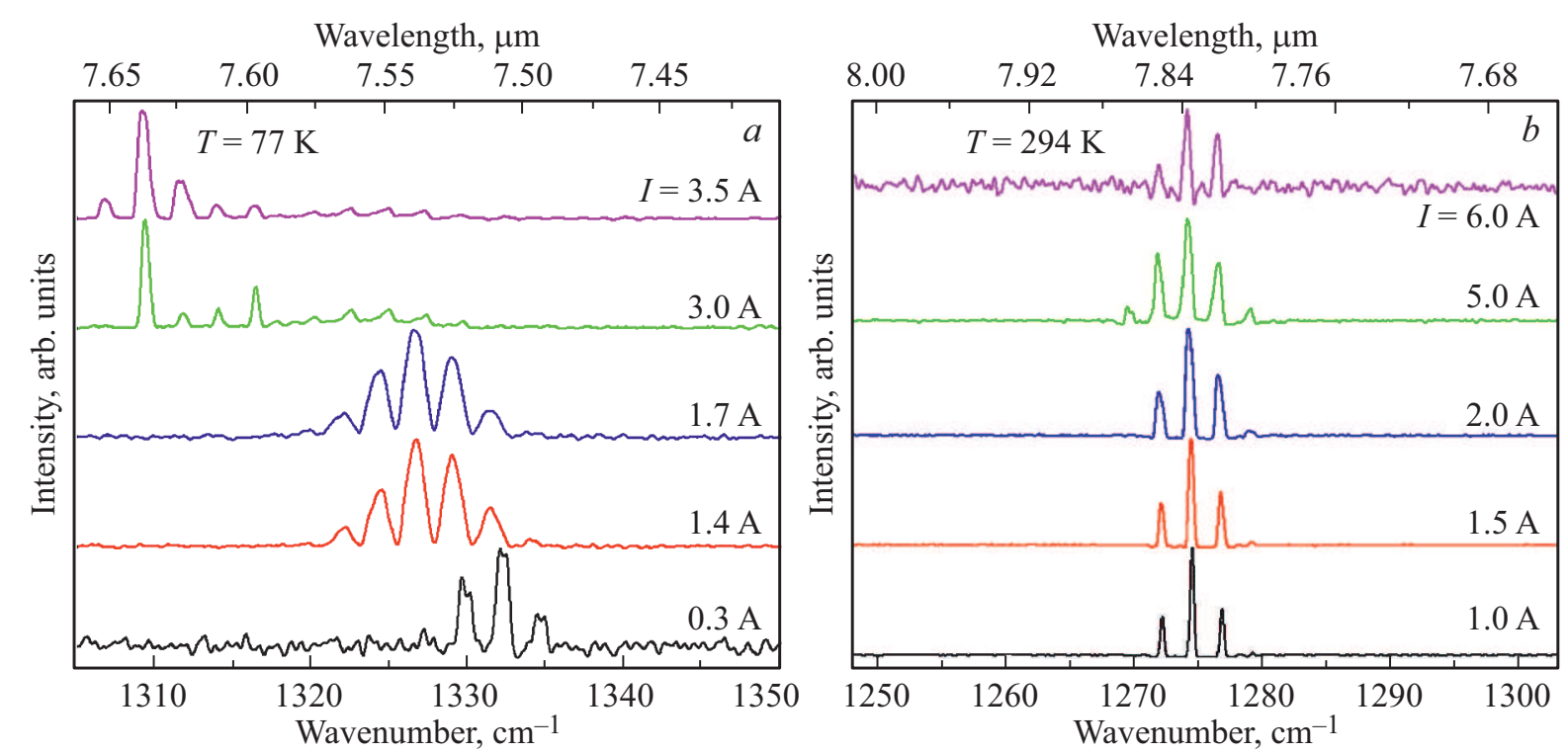

Рис. 1. Нормированные спектры поверхностной генерации исследуемого ККЛ. $a$ - температура 77 К, различные уровни токовой накачки (данные при токах накачки до 1.7 А представлены в работе [17]); $b$ - температура $294 \mathrm{~K}$, различные уровни токовой накачки.

Оптикс на промышленной установке молекулярнопучковой эпитаксии Riber 49 [11,12]. Использована конструкция волновода с верхней обкладкой толщиной 3.9 мкм на основе фосфида индия (уровень легирования $\left.n=1.0 \cdot 10^{17} \mathrm{~cm}^{-3}\right)$. Активная область сформирована на основе гетеропары твердых растворов $\mathrm{In}_{0.53} \mathrm{Ga}_{0.47} \mathrm{As} / \mathrm{Al}_{0.48} \mathrm{In}_{0.52} \mathrm{As}$ с двухфононным опустошением нижнего уровня в каскаде $[13,14]$. В качестве контактных слоев использованы слои $\mathrm{In}_{0.53} \mathrm{Ga}_{0.47} \mathrm{As}$ толщиной 100 и 20 нм с уровнями легирования $1.0 \cdot 10^{17}$ и $1.0 \cdot 10^{19} \mathrm{~cm}^{-3}$ соответственно. Формирование кристалла ККЛ проводилось по методике, аналогичной описанной ранее [15]. Использована конструкция глубокой мезы с затравом в подложку InP. Внешний радиус кольцевого резонатора составил 201 мкм. Ширина резонатора вблизи поверхности составила 20 мкм. Монтаж лазерного кристалла проводился подложкой вниз на медный теплоотвод при помощи индиевого припоя.

Травление окон, через которые осуществлялся поверхностный вывод излучения, проводилось в сверхвысоком вакууме фокусированным пучком ионов галлия с энергией 30 кэВ при рабочем токе 490 пА [16]. Ионная доза при травлении штрихов дифракционной решетки составляла $4.5 \cdot 10^{10} \Pi К л / \mathrm{cm}^{2}$. В ходе исследований был выбран режим травления, обеспечивающий формирование субволнового рельефа на вытравленной поверхности, что должно было привести к повышению эффективности вывода излучения. Окна с текстурированным слоем имели форму правильной трапеции с периодом следования в угловых координатах $18.9^{\circ}$. Глубина травления слоев гетероструктуры составила $\sim 200 \mathrm{HM}$, что превышало суммарную толщину контактных слоев $\operatorname{In}_{0.53} \mathrm{Ga}_{0.47} \mathrm{As}$. Полученное методом сканирующей электронной микроскопии изображение ККЛ в конструкции с кольцевым резонатором с 9 вытравленными окнами представлено ранее в работе [17].

Спектры стимулированного излучения измерялись в режиме пошагового сканирования с помощью фурьеспектрометра Bruker Vertex 80v при криогенных температурах и с помощью фурье-спектрометра Bruker Vertex 70v при температуре 294 К. Детектирование оптического сигнала осуществлялось с помощью фотодиода $\mathrm{HgCdTe}$. Спектральное разрешение составляло $0.2 \mathrm{~cm}^{-1}$. При исследовании спектров излучения длительность импульсов тока накачки составляла $\tau=200 \mathrm{Hc}$, а при исследовании вольт-, ватт-амперных характеристик $\tau=70$ нс. Частота следования импульсов $f$ составила 10 кГц для измерений при температуре $77 \mathrm{~K}$ и 25 кГц для измерений при температуре 294 К. Исследование распределения интенсивности излучения ККЛ в зоне дальнего поля проведено при токе накачки 0.92 А. Для регистрации распределения интенсивности излучения использован фотоприемник Vigo PVI-4TE-10.6, область видимости детектора (FOW) составляла $36^{\circ}$.

\section{3. Результаты и их обсуждение}

Спектры поверхностной лазерной генерации, измеренные при температуре $77 \mathrm{~K}$ и различных уровнях токовой накачки $I$, представлены на рис. $1, a$. При пороговом токе $I=I_{\text {th }}=0.17$ А наблюдается генерация вблизи 7.5 мкм, спектр представлен тремя оптическими модами. Межмодовое расстояние $\Delta \lambda$ соответствует случаю мод шепчущей галереи, для которых $\Delta \lambda=\lambda^{2} /\left(2 \pi R_{\text {out }}\right) n_{g}$, где $n_{g}-$ групповой коэффициент преломления, $R_{\text {out }}-$ внешний радиус кольцевого резонатора, $\lambda$ - длина волны излучения. С ростом уровня накачки до $8 I_{\text {th }}$ наблюдается 
длинноволновый сдвиг. При превышении $17 I_{\text {th }}$ в спектре генерации возникает дополнительная группа оптических мод вблизи 7.62 мкм с подавлением интенсивности группы мод вблизи 7.55 мкм. Увеличение температуры приводит к длинноволновому сдвигу, что может быть обусловлено уменьшением разрыва зоны проводимости на гетерогранице [18,19]. При температуре $294 \mathrm{~K}$ лазерная генерация наблюдается вблизи длины волны 7.85 мкм (см. рис. $1, b)$, как и для случая ККЛ с поверхностным выводом излучения через дифракционную решетку 2-го порядка [20]. Спектр генерации вблизи порога представлен 3 оптическими модами. По величине межмодового расстояния получена оценка: $n_{g}=3.4$. Повышение уровня накачки до $6 I_{\text {th }}$ не приводит к изменению модового состава, а также сдвигу спектрального положения мод. На основе информации о значении пороговой плотности тока $j_{\text {th }}$, а также величины $g \Gamma=3.3 \mathrm{~cm} /$ кА [21], где $g$ дифференциальное усиление, $\Gamma$ - фактор оптического ограничения, проведена оценка полных оптических потерь в исследуемом лазере: $\alpha_{\text {Ring }}=j_{\text {th }} g \Gamma \sim 10 \mathrm{~cm}^{-1}$. На основе выражения $Q=2 \pi n_{g} /\left(\lambda \alpha_{\text {Ring }}\right)$, представленного ранее [22], произведена оценка добротности резонатоpa: $Q \approx 2600$.

Проведено исследование зависимости интегральной интенсивности излучения ККЛ от тока накачки, измеренной по нормали к плоскости образца (см. рис. 2). Измерения проведены при температуре 294 К. Пороговая плотность тока $j_{\text {th }}$ составила $3.2 \kappa \mathrm{A} / \mathrm{cm}^{2}$. Показана лазерная генерация с увеличением уровня токовой накачки до $8 I_{\text {th. }}$.

На основе информации о величине $j_{\text {th }}$ при различных температурах $T$ и выражения $j_{\text {th }}(T)=j_{0} \exp \left(T / T_{0}\right)$

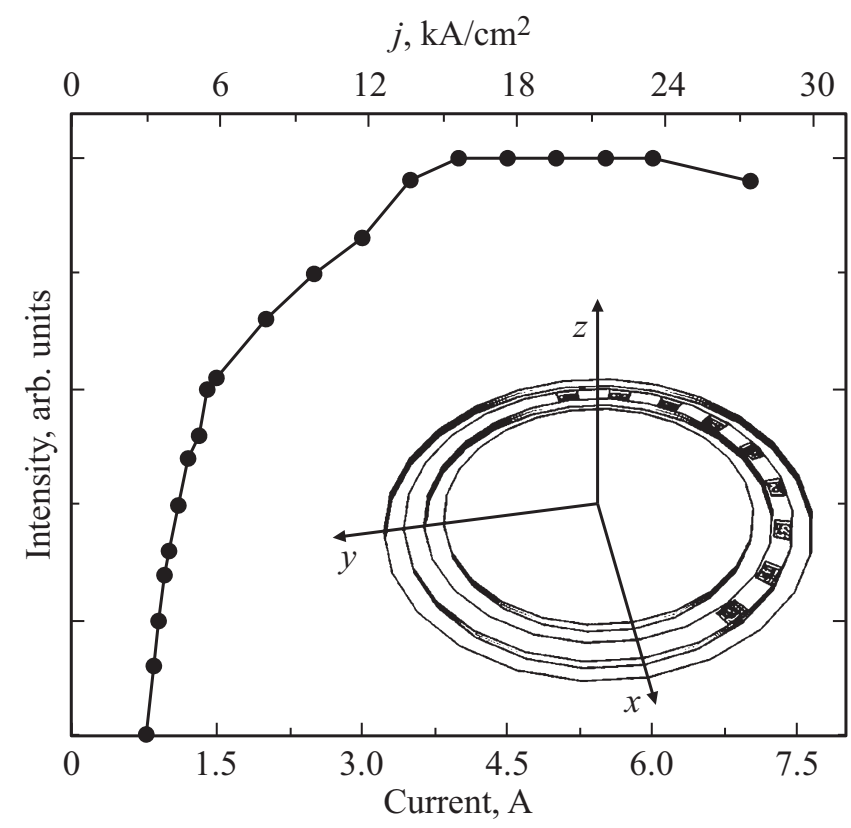

Рис. 2. Зависимость интегральной интенсивности излучения ККЛ от тока накачки при температуре 294 К. На вставке схематически показана конструкция кольцевого резонатора.
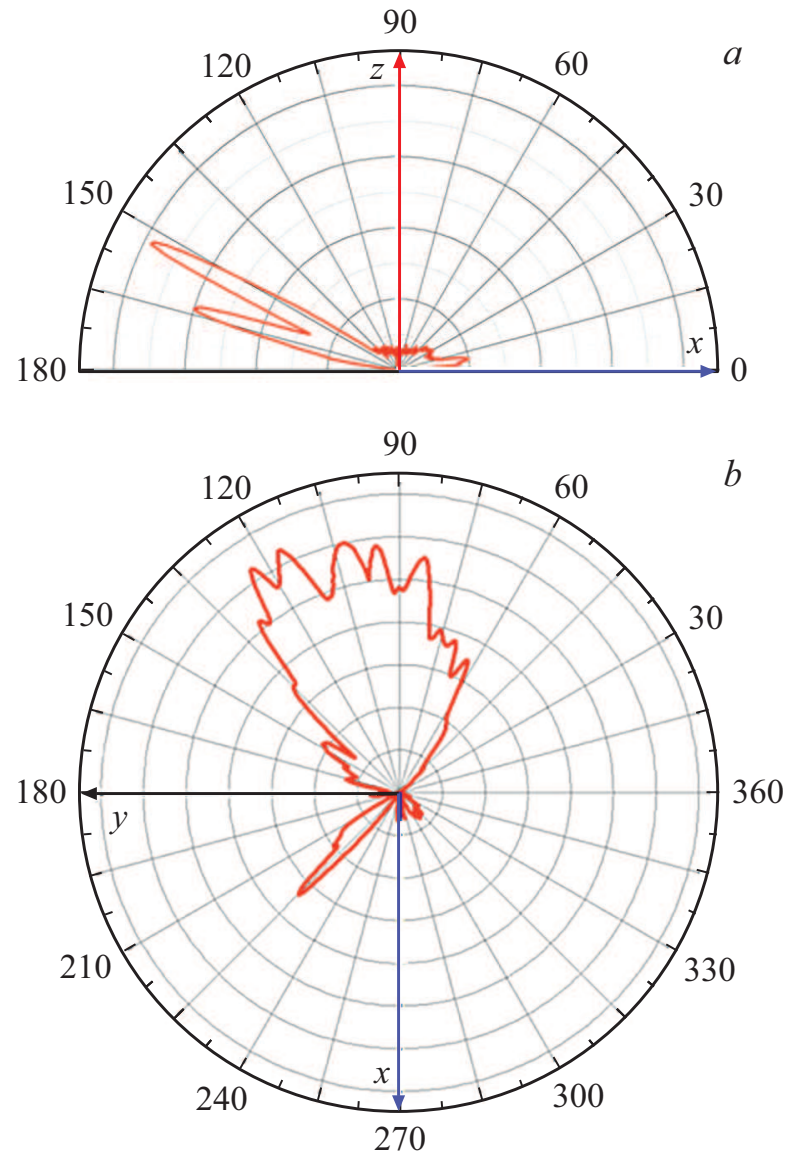

Pис. 3. Диаграммы направленности в плоскости $x z$ (a) и в плоскости $x y(b)$. (Цветной вариант рисунка представлен в электронной версии статьи).

проведена оценка характеристической температуры стабильности порогового тока, которая составила $T_{0}=149 \mathrm{~K}$. Оценочная плотность порогового тока при нулевой температуре составляет $j_{0}=0.4 \kappa \mathrm{A} / \mathrm{cm}^{2}$.

Проведено исследование распределения интенсивности излучения ККЛ в зоне дальнего поля. Показано, что сформированные окна, содержащие рельефные слои, позволили осуществить направленный поверхностный вывод излучения из кольцевого ККЛ. В плоскости $x z$ диаграмма направленности имеет 2 максимума с углами вывода $\sim 7^{\circ}$ (см. вставку на рис. 3,a). Наличие двух максимумов можно объяснить тем, что излучение в кольцевом резонаторе может распространяться в двух взаимно противоположных направлениях. Вывод излучения в плоскости $x z$ осуществляется в диапазоне углов $\sim(63-75)^{\circ}$, отсчитанных от нормали к плоскости резонатора (рис. $3, b)$.

\section{4. Заключение}

В ходе исследований представлены результаты по формированию и изучению характеристик поверхностно-излучающих ККЛ спектрального диапазона 
7.5-8.0 мкм с кольцевым резонатором, с выводом излучения через окна, содержащие текстурированный слой, сформированный в верхней обкладке волновода методом прямой литографии сфокусированным ионным пучком. Показано, что сформированные окна позволили осуществить направленный поверхностный вывод излучения из кольцевого ККЛ. Распределение интенсивности излучения ККЛ в зоне дальнего поля в плоскости $x z$ показывает, что излучение выводится через окна в диапазоне углов $\sim(63-75)^{\circ}$, отсчитанных от нормали к плоскости резонатора.

\section{Финансирование работы}

Работа выполнена при финансовой поддержке гранта Российского научного фонда (проект № 20-79-10285).

\section{Конфликт интересов}

Авторы заявляют, что у них нет конфликта интересов.

\section{Список литературы}

[1] R. Szedlak, T. Hisch, B. Schwarz, M. Holzbauer, D. MacFarland, T. Zederbauer, H. Detz, A.M. Andrews, W. Schrenk, S. Rotter, G. Strasser. Sci. Rep., 8 (1), 7998 (2018).

[2] B. Hinkov, J. Hayden, R. Szedlak, P. Martin-Mateos, B. Jerez, P. Acedo, G. Strasser, B. Lendl. Opt. Express, 27 (10), 14716 (2019).

[3] H. Knötig, B. Hinkov, R. Weih, S. Höfling, J. Koeth, G. Strasser. Appl. Phys. Lett., 116 (13), 131101 (2020).

[4] D.H. Wu, M. Razeghi. APL Mater., 5 (3), 035505 (2017).

[5] Y. Bai, S. Tsao, N. Bandyopadhyay, S. Slivken, Q.Y. Lu, D. Caffey, M. Pushkarsky, T. Day, M. Razeghi. Appl. Phys. Lett., 99 (26), 261104 (2011).

[6] E. Mujagić, L.K. Hoffmann, S. Schartner, M. Nobile, H. Detz, D. Andrijasevic, M. Austerer, W. Schrenk, A.M. Andrews, P. Klang, M.P. Semtisiv, W.T. Masselink, G. Strasser. Proc. SPIE, 7230, 723015 (2009). doi: 10.1117/12.808466

[7] A.O. Dirisu, G. Silva, Z. Liu, C.F. Gmachl, F.J. Towner, J. Bruno, D.L. Sivco. IEEE Photon. Technol. Lett., 19 (4), 221 (2007).

[8] B. Päivänranta, T. Saastamoinen, M. Kuittinen. Nanotechnology, 20 (37), 375301 (2009).

[9] A. Deinega, I. Valuev, B. Potapkin, Y. Lozovik. J. Opt. Soc. Am. A, 28 (5), 770 (2011).

[10] J.A. Dobrowolski, D. Poitras, P. Ma, H. Vakil, M. Acree. Appl. Optics, 38 (16), 3075 (2002).

[11] A.V. Babichev, A.G. Gladyshev, A.S. Kurochkin, E.S. Kolodeznyi, G.S. Sokolovskii, V.E. Bougrov, L.Ya. Karachinsky, I.I. Novikov, A.G. Bousseksou, A.Yu. Egorov. Semiconductors, 52 (8), 1082 (2018).

[12] A.V. Babichev, V.V. Dudelev, A.G. Gladyshev, D.A. Mikhailov, A.S. Kurochkin, E.S. Kolodeznyi, V.E. Bougrov, V.N. Nevedomskiy, L.Ya. Karachinsky, I.I. Novikov, D.V. Denisov, A.S. Ionov, S.O. Slipchenko, A.V. Lutetskiy, N.A. Pikhtin, G.S. Sokolovskii, A.Yu. Egorov. Semiconductors, 45 (7), 735 (2019).
[13] A.V. Babichev, A.S. Kurochkin, E.C. Kolodeznyi, A.V. Filimonov, A.A. Usikova, V.N. Nevedomsky, A.G. Gladyshev, L.Ya. Karachinsky, I.I. Novikov, A.Yu. Egorov. Semiconductors, 52 (6), 745 (2018).

[14] A.V. Babichev, A.G. Gladyshev, A.S. Kurochkin, V.V. Dudelev, E.S. Kolodeznyi, G.S. Sokolovskii, V.E. Bugrov, L.Ya. Karachinsky, I.I. Novikov, D.V. Denisov, A.S. Ionov, S.O. Slipchenko, A.V. Lyutetskii, N.A. Pikhtin, A.Yu. Egorov. Techn. Phys. Lett., 45 (4), 398 (2019).

[15] V.V. Dudelev, D.A. Mikhailov, A.V. Babichev, A.D. Andreev, S.N. Losev, E.A. Kognovitskaya, Yu.K. Bobretsova, S.O. Slipchenko, N.A. Pikhtin, A.G. Gladyshev, D.V. Denisov, I.I. Novikov, L.Ya. Karachinsky, V.I. Kuchinskii, A.Yu. Egorov, G.S. Sokolovskii. Quant. Electron., 50 (2), 141 (2020).

[16] A.V. Babichev, D.A. Pashnev, A.G. Gladyshev, D.V. Denisov, G.V. Voznyuk, L.Ya. Karachinsky, I.I. Novikov, M.I. Mitrofanov, V.P. Evtikhiev, D.A. Firsov, L.E. Vorob'ev, N.A. Pikhtin, A.Yu. Egorov. Techn. Phys. Lett., 46 (4), 312 (2020).

[17] A.V. Babichev, E.S. Kolodeznyi, A.G. Gladyshev, D.V. Denisov, G.V. Voznyuk, D.I. Kuritsyn, M.I. Mitrofanov, S.O. Slipchenko, A.V. Lyutetskii, V.P. Evtikhiev, L.Ya. Karachinsky, I.I. Novikov, N.A. Pikhtin, S.V. Morozov, A.Yu. Egorov. Semiconductors, 54 (14), 1816 (2020).

[18] Y. Bai, S. Slivken, S. Kuboya, S.R. Darvish, M. Razeghi. Nature Photonics, 4 (2), 99 (2010).

[19] A.V. Babichev, A.G. Gladyshev, D.V. Denisov, L.Ya. Karachinsky, I.I. Novikov, L. Boulley, A. Bousseksou, N.A. Pikhtin, A.Yu. Egorov. Opt. Spectrosc., 127 (2), 279 (2019).

[20] A.V. Babichev, E.S. Kolodeznyi, A.G. Gladyshev, D.V. Denisov, G.V. Voznyuk, M.I. Mitrofanov, N.Yu. Kharin, V.Yu. Panevin, S.O. Slipchenko, A.V. Lyutetskii, V.P. Evtikhiev, L.Ya. Karachinsky, I.I. Novikov, N.A. Pikhtin, A.Yu. Egorov. Semiconductors, 55 (7), 822 (2021).

[21] A.V. Babichev, D.A. Pashnev, A.G. Gladyshev, A.S. Kurochkin, E.S. Kolodeznyi, L.Ya. Karachinsky, I.I. Novikov, D.V. Denisov, V.V. Dudelev, G.S. Sokolovskii, D.A. Firsov, L.E. Vorob’ev, S.O. Slipchenko, A.V. Lutetskiy, N.A. Pikhtin, A.Yu. Egorov. Opt. Spectrosc., 128 (8), 1187 (2020).

[22] J. Faist, C. Gmachl, M. Striccoli, C. Sirtori, F. Capasso, D.L. Sivco, A.Y. Cho. Appl. Phys. Lett., 69 (17), 2456 (1996).

Редактор Л.В. Шаронова 


\section{Quantum-cascade laser with radiation output through a textured layer}

A.V. Babichev ${ }^{1}$, E.S. Kolodeznyi ${ }^{1}$, A.G. Gladyshev ${ }^{1}$, D.V. Denisov ${ }^{2}$, G.V. Voznyuk ${ }^{3}$, M.I. Mitrofanov ${ }^{3}$, D.A. Mikhailov ${ }^{3}$, D.V. Chistyakov ${ }^{3}$, D.I. Kuritsyn ${ }^{4}$, V.V. Dudelev ${ }^{3}$, S.O. Slipchenko ${ }^{3}$, A.V. Lyutetskii ${ }^{3}$, V.P. Evtikhiev ${ }^{3}$, L.Ya. Karachinsky ${ }^{1,3,5}$, I.I. Novikov ${ }^{1,3,5}$, S.V. Morozov ${ }^{4}$, G.S. Sokolovskii ${ }^{3}$, N.A. Pikhtin ${ }^{3}$, A.Yu. Egorov ${ }^{1}$

${ }^{1}$ ITMO University, 197101 St. Petersburg, Russia

2 St. Petersburg Electrotechnical University „LETl“, 197022 St. Petersburg, Russia

${ }^{3}$ loffe Institute,

194021 St. Petersburg, Russia

${ }^{4}$ Institute for Physics of Microstructures,

Russian Academy of Sciences, 603950 Nizhny Novgorod, Russia

${ }^{5}$ Connector Optics LLC,

194292 St. Petersburg, Russia

Abstract The results of studies of ring quantum-cascade laser with a surface emission with radiation output through a textured layer formed in the layers of the top cladding of the waveguide by ultrahigh vacuum ion beam milling are presented. The far-field profile shows that the radiation is output through the windows with a textured layer in the range of angles of $\sim(63-75)^{\circ}$ to the normal. 